\title{
Evaluation of Motion Blur Considering Temporal Frequency Characteristics of Video Camera and LCD Systems
}

\author{
Seok-Min CHAE ${ }^{\dagger}$, In-Ho SONG ${ }^{\dagger a}$, Sung-Hak LEE ${ }^{\dagger}$, Nonmembers, and Kyu-Ik SOHNG ${ }^{\dagger}$, Member $^{-}$
}

SUMMARY In this study, we show that the motion blur is caused by exposure time of video camera as well as the characteristics of LCD system. Also, we suggest that evaluation method of motion picture quality according to the frequency response of video camera and LCD systems of hold and scanning backlight type.

key words: motion blur, LCD systems, scanning backlight

\section{Introduction}

In this study, we give a modeling method for the frequency characteristics of LCDs. Generally motion blur has been analyzed based on LCD parameters such as the response time and backlight type but the motion blurring phenomenon was ultimately attributed to the characteristics of the video camera. When using a video camera, exposure time is determined according to the condition of peripheral environments. At the same time, some blurring occurs on the video signal itself due to the integral calculus of the exposure time. Therefore, to analyze how this exposure time influences the motion blur, we also analyzed the frequency characteristics of the video camera. To generalize the evaluation model, first the entire frequency characteristics were analyzed when the video image was presented on an LCD monitor. Then, we analyzed two kinds of LCD products commercialized actually with the result of our model. Recent LCD systems have been used to enhance the quality of motion images such as the doubled frame rate method and scanning (blinking) backlight. In this study, we compared and analyzed the characteristics of motion blur on commercial LCDs, produced with the doubling frame rate and blinking backlight method.

\section{Analysis of Blurring Effects in LCD}

Generally the blurring on the motion images on LCD has been known to occur as a result of slow response time. However, even if response time is $0 \mathrm{~ms}$, blurring can occur because of the existing hold type [1], [2]. So, several methods have been presented. These are inserting black data between every two input frames [3], flashing backlight [4], doubling the frame rate [5], and motion compensated inverse filtering [6]. The two ways adopted a blinking-type method and

Manuscript received November 9, 2011.

Manuscript revised January 21, 2012.

${ }^{\dagger}$ The authors are with School of Electrical Engineering and Computer Science, Kyungpook National University, Korea.

a) E-mail: songinho@ee.knu.ac.kr

DOI: 10.1587/transinf.E95.D.1823 the last two ways adopted a hold-type method. Recently, a system combining the above two types has been applied.

Figure 1 shows the optical output pattern of these two types of LCD system. The response time of the liquid crystal and the $100 \%$ duty pulse width influenced the frequency characteristics in the hold-type LCD. In the blinking-type LCD, we turned on the backlight after the transmittance of liquid crystal is the peak. The response time did not significantly influence the frequency characteristics but the response time for switching backlight and the pulse width of backlight did influence the frequency characteristics. Therefore, from the evaluation of the pulse width and the response time of liquid crystal and response time in switching the backlight, the entire frequency characteristics of LCD system could be determined. First, the frequency characteristics for the pulse width of lighting could be analyzed from the aperture effect. Secondly, the response time of the liquid crystal and the response time in switching backlight could be modeled as a LPF. Therefore, the frequency characteristics for the output signal of LCD could be presented as below [1], [2].

$$
\begin{aligned}
& G_{L C D}(f)=G_{L C D_{1}}(f) \times G_{L C D_{2}}(f) \\
& G_{L C D_{1}}(f)=\left|\frac{\sin \left(\pi f t_{0}\right)}{\pi f t_{0}}\right| \\
& G_{L C D_{2}}(f)=\sqrt{\frac{1}{1+(2 \pi \tau f)^{2}}}
\end{aligned}
$$

where $G_{L C D}(f)$ means the entire signal gain by frequency, $G_{L C D 1}(f)$ means the signal gain by frequency for the pulse width of backlight and $G_{L C D 2}(f)$ means the signal gain by frequency for the different response time of LCD and switching backlight. Also, $f$ means temporal frequency, $t_{0}$ is the pulse width of the backlight, and $\tau$ is the time constant calculated from the response time of liquid crystal or the response time of backlight switching.

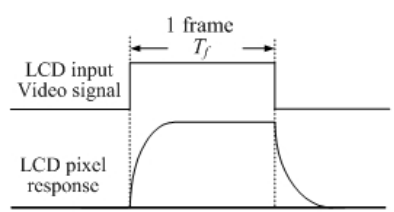

(a)

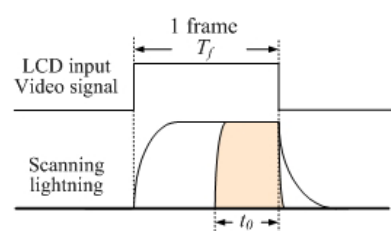

(b)
Fig. 1 The output signal of LCD: (a) Traditional hold-type backlight; (b) scanning backlight. 


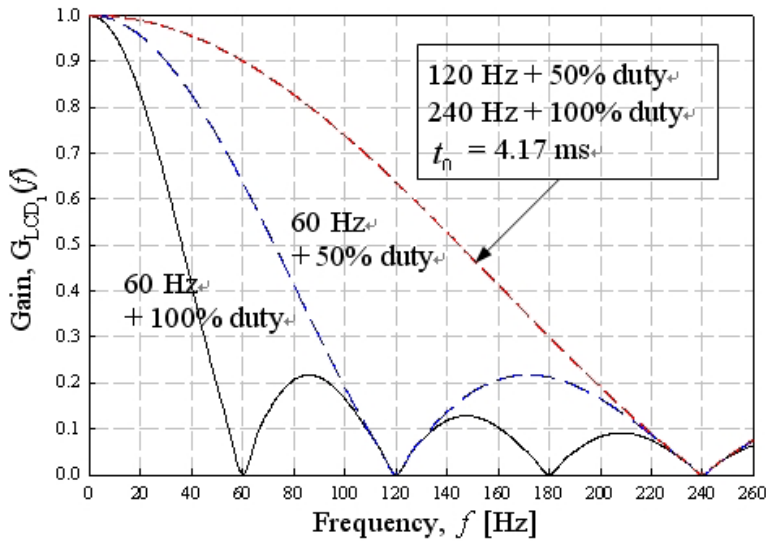

(a)

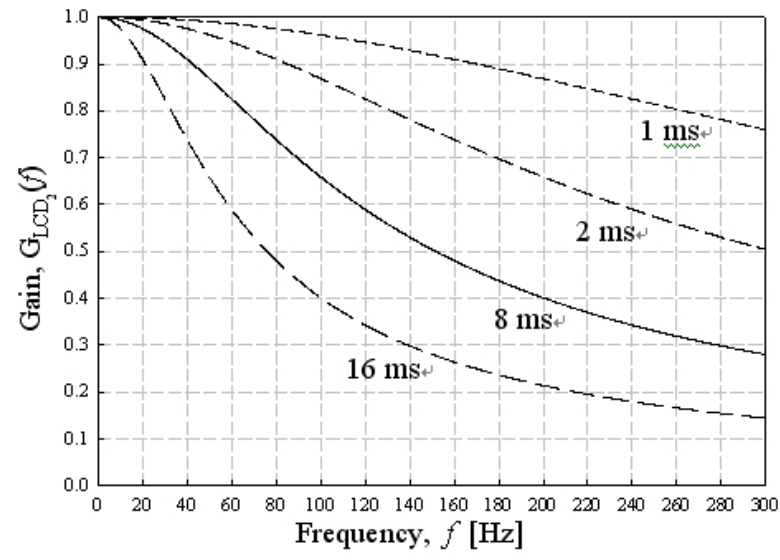

(b)

Fig. 2 Characteristic spectrums of factors to evaluate blurring effects in LCDs: (a) Frequency response $G_{L C D 1}(f)$ by different duty ratios; (b) frequency response $G_{L C D 2}(f)$ by LCD or backlight switching.

Figure 2 shows the characteristic spectrums of factors for evaluating blurring effects in LCDs.

\section{Analysis of Temporal Frequency Characteristics in Video Camera Systems}

Many researchers have studied motion blur in LCD displays, they mainly focus on LCD devices. However, the video signal itself captured by a camera also has an effect on motion blurs. The shutter speed of the camera is determined according to the peripheral brightness of the environment and the iris of camera lens when shooting. For this procedure, temporal integration has been done for a while. This means that when you shoot moving objects with a fixed camera or fixed objects with a moving camera, motion blur occurs due to the integral calculus for the exposure time. In this study, we suggest a motion blur model based on the exposure time of video cameras. $g_{s}(t)$ is sampled by an ideal impulse from $g(t)$ and Fourier transform of $g_{s}(t)$ are given as Eq. (4) and (5), respectively.

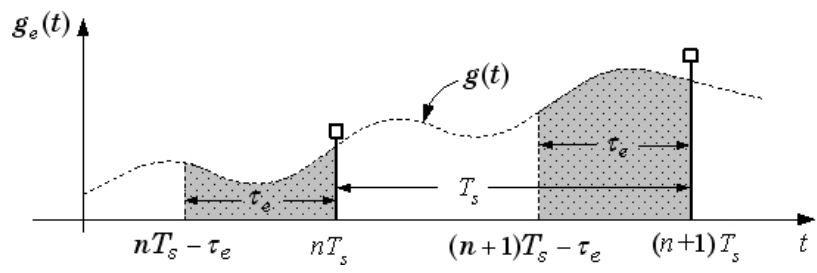

Fig. 3 Sampling method of the video camera.

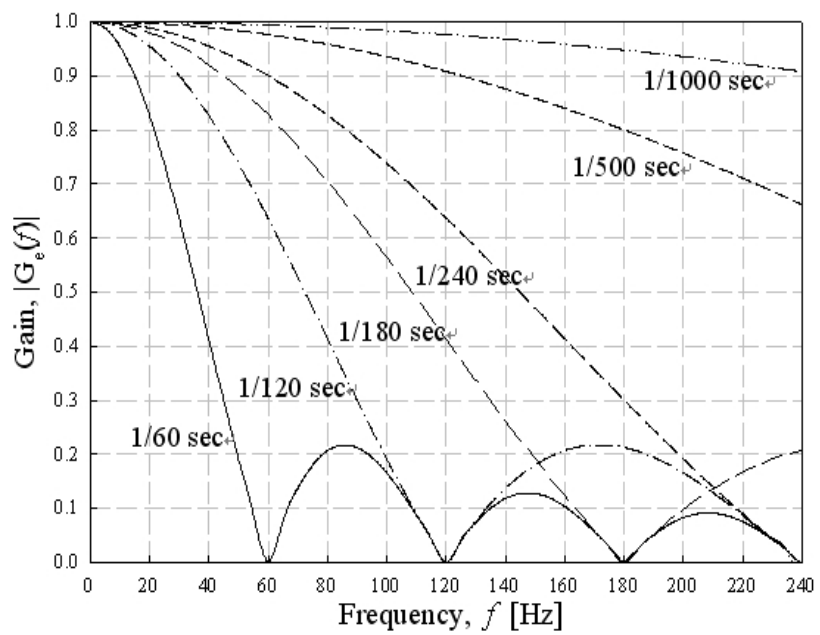

Fig. 4 Characteristic spectrums of video signals with various exposure times.

$$
\begin{aligned}
& g_{s}(t)=g(t) \cdot \sum_{n} \delta\left(t-n T_{s}\right) \\
& G_{s}(f)=f_{s} \sum_{m} G\left(f-m f_{s}\right)
\end{aligned}
$$

Figure 3 shows sampling data sequence integrated in a video camera. CCD video camera outputs sampled the signal every frame period $T_{s}$. This sampled signal is the integrated signal for the exposure time $\tau_{e}$ according to the shutter speed. This output signal $g_{e}(t)$ would be

$$
\begin{aligned}
g_{e}(t) & =\sum_{n}\left(\int_{n T_{s}-\tau_{e}}^{n T} g(t) d t\right) \cdot \delta\left(t-n T_{s}\right) \\
& =\sum_{n}\left(\lim _{\Delta \tau \rightarrow 0} \sum_{k=0}^{\tau_{e}} g(t-k \Delta \tau)\right) \cdot \delta\left(t-n T_{s}\right)
\end{aligned}
$$

$g_{e}(t)$ becomes following equations by the shifting theorem of Fourier transform.

$$
\begin{aligned}
G_{e}(f) & =G_{s}(f) \cdot \lim _{\Delta \tau \rightarrow 0}\left(\sum_{k=0}^{\tau_{e} / \Delta \tau} e^{-j 2 \pi f k \Delta \tau}\right) \\
& =G_{s}(f) \int_{0}^{\tau_{e}} e^{-j 2 \pi f \tau} d \tau \\
& =G_{s}(f) \cdot \tau_{e} \cdot \frac{\sin \left(\pi f \tau_{e}\right)}{\pi f \tau_{e}} \cdot e^{-j \pi f \tau_{e}}
\end{aligned}
$$

Therefore, the normalized temporal frequency characteristics of video camera $\left|G_{e}(f)\right|$ is given by a following 
equation.

$$
\left|G_{e}(f)\right|=\left|\frac{\sin \left(\pi f \tau_{e}\right)}{\pi f \tau_{e}}\right|
$$

Where, $\left|G_{e}(f)\right|$ means the signal gain by the frequency according to the shutter speed of camera, $\tau_{e}$ means the exposure time, and $f$ means the temporal frequency.

The frequency characteristics of the video camera are presented according to the exposure time in Fig. 4. As the exposure time is increased, high frequency gain is reduced. Generally, the video signal is produced with a shutter speed of $1 / 60 \mathrm{sec}$ or $1 / 100 \mathrm{sec}$ according to the frequency of a lighting of electric power source when filming TV station broadcasting studios. Therefore, the video signal corresponding to the part of high frequency is reduced considerably due to slow shutting speed. Of course, a video signal with high shutter speed or a caption inserted in the video edition process is an impulsive signal, and motion blur can hardly occur. In that case, the frequency characteristics can be only considered for the LCD display itself.

\section{Results of Blurring Effects Considering the Video Camera, Backlight, and LCD}

The overall frequency characteristics can express the multiplication of the frequency characteristics $G_{\text {total }}(f)$ of a camera and an LCD system as follows.

$$
G_{\text {total }}(f)=G_{e}(f) \times G_{L C D}(f)
$$

However, captions and graphic images can be analyzed only for the effect on LCDs, $G_{L C D}(f)$.

We compared and analyzed the frequency characteristics for hold type backlight and scanning backlight of LCD TVs. In these TVs, the method of doubling frame rate was used. One has $240 \mathrm{~Hz}$ frame-rate and a $100 \%$ duty holdtype backlight and the other has $120 \mathrm{~Hz}$ frame-rate and a $50 \%$ duty scanning backlight. Input video signals for test are captured by exposure time of $1 / 60 \mathrm{sec}$ and $1 / 100 \mathrm{sec}$. We measured the response time of the LCDs and the backlight switching time. The results of the measurement are shown in Fig. 5. LCD response time is about $4.5 \mathrm{~ms}$ and LED switching time is $1 \mathrm{~ms}$, respectively. Using these data

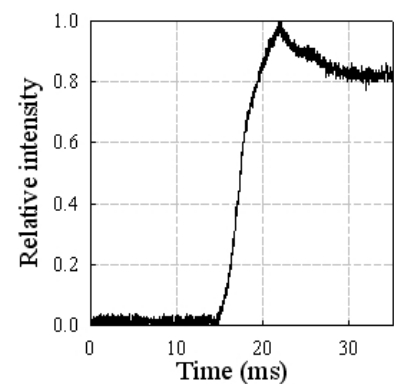

(a)

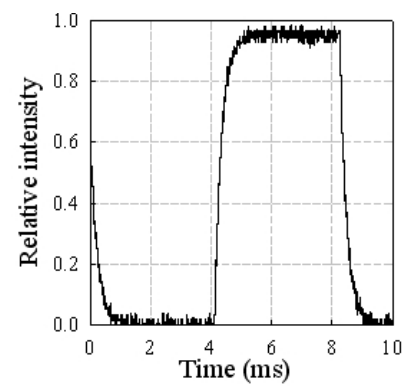

(b)
Fig.5 Measured response time used for the comparison: (a) The response time of the LCD; (b) response time of LED backlight switching. and Eq. (9), the total frequency characteristics of each LCD TV are calculated and shown in Fig. 6. It shows that the frequency characteristics of the two types of LCD TV will be different from each other. On the other hand, in case of

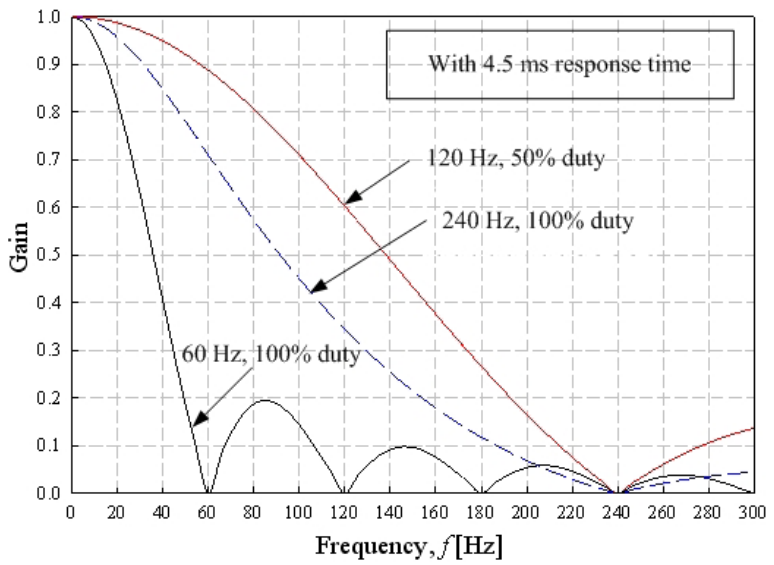

(a)

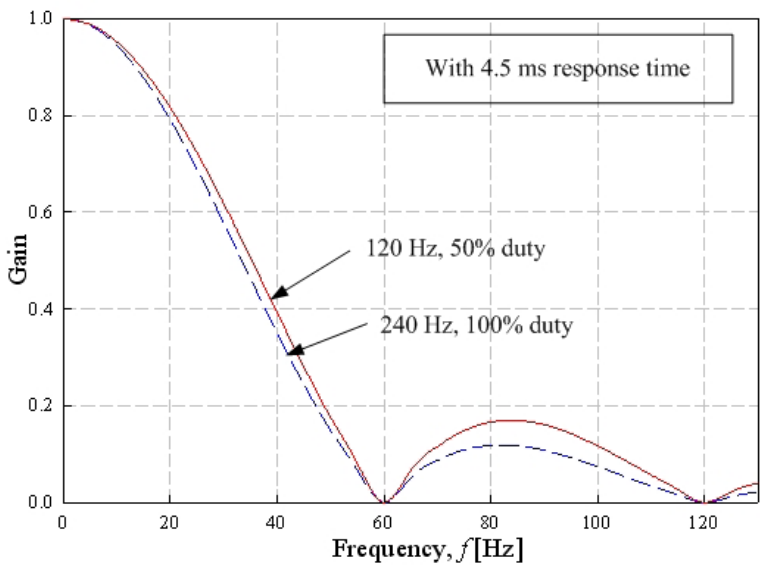

(b)

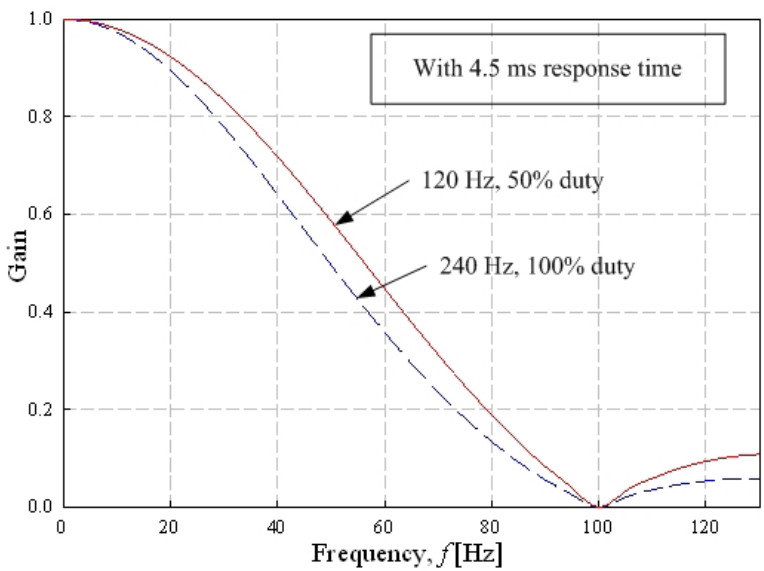

(c)

Fig. 6 Comparisons of the frequency response characteristics for different frame rates and backlight duties: (a) By ideal impulsive video signal; (b) video signal shot by $1 / 60 \mathrm{sec}$ shutter speed; (c) video signal shot by $1 / 100$ sec shutter speed. 


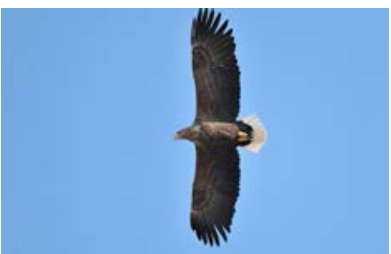

(a)

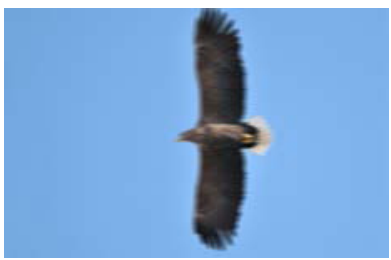

(c)

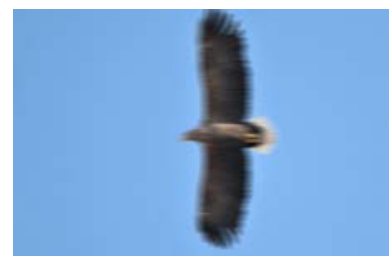

(b)

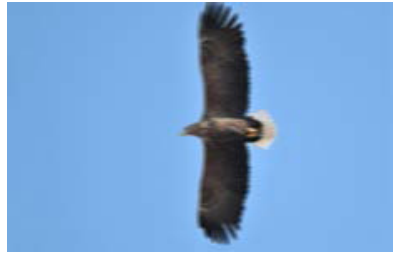

(d)
Fig. 7 Comparisons of the image for different frame rates and backlight duties: (a) Original still image; (b) $60 \mathrm{~Hz}, 100 \%$ duty, $4.5 \mathrm{~ms}$ LCD response time by $1 / 60 \mathrm{sec}$ shutter speed; (c) $240 \mathrm{~Hz}, 100 \%$ duty, $4.5 \mathrm{~ms} \mathrm{LCD}$ response time by $1 / 60 \mathrm{sec}$ shutter speed; (d) $120 \mathrm{~Hz}, 50 \%$ duty, $1 \mathrm{~ms}$ LED scanning backlight response time by $1 / 60 \mathrm{sec}$ shutter speed.

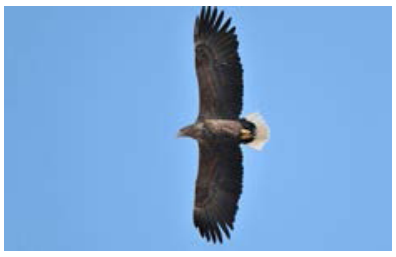

(a)

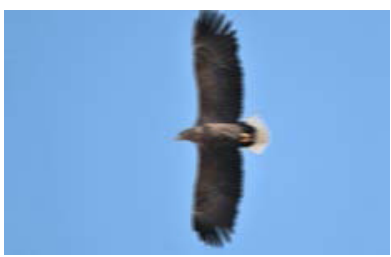

(c)

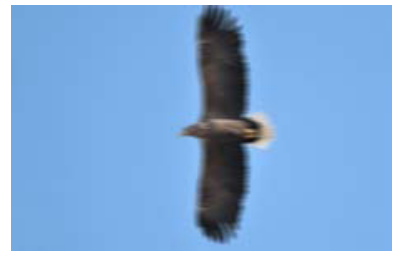

(b)

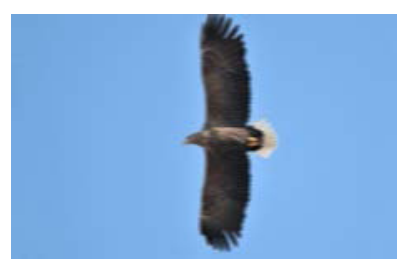

(d)
Fig. 8 Comparisons of the image for different frame rates and backlight duties: (a) Original still image; (b) $60 \mathrm{~Hz}, 100 \%$ duty, $4.5 \mathrm{~ms} \mathrm{LCD}$ response time by $1 / 100 \mathrm{sec}$ shutter speed; (c) $240 \mathrm{~Hz}, 100 \%$ duty, $4.5 \mathrm{~ms}$ LCD response time by $1 / 100 \mathrm{sec}$ shutter speed; (d) $120 \mathrm{~Hz}, 50 \%$ duty, $1 \mathrm{~ms}$ LED scanning backlight response time by $1 / 100 \mathrm{sec}$ shutter speed.

displaying the video shot by slow shutter speed, the performance difference between the two types of TV is reduced. If we display the video shot by high shutter speed or produced by computer, we can significantly recognize the difference. From the results of comparing the $240 \mathrm{~Hz}$ frame-rate with $100 \%$ hold-type backlight and the $120 \mathrm{~Hz}$ frame-rate with $50 \%$ duty scanning backlight, it is assumed that $120 \mathrm{~Hz}$ frame-rate with $50 \%$ duty scanning backlight is slightly superior.

We performed simulation considering the frequency characteristics of the shutter speed of the camera and the response time of the LCD system. Figure 7 and Fig. 8 show the still image for the different frame rate and backlight duty in $1 / 60 \mathrm{sec}$ and $1 / 100 \mathrm{sec}$ shutter speed respectively. In this simulation, the moving object speed was assumed to be 1800 pixels/sec and the shutter speed of the camera was $1 / 60 \mathrm{sec}$ and $1 / 100 \mathrm{sec}$. We were moveing the still image moves as the moving object speed (1800 pixels/sec) and simulatied the image for each LCD conditions and shutter speeds. We can be confirmed that the motion blur of the $120 \mathrm{~Hz}, 50 \%$ duty scanning backlight LCD system is less than other LCD systems.

Therefore, the $120 \mathrm{~Hz}, 50 \%$ duty scanning backlight LCD system is shown to be more effective than the other kind of backlight even though having higher frame frequency of the $240 \mathrm{~Hz}$ or more in the case of the $4.5 \mathrm{~ms}$ response time of LCDs. And the $120 \mathrm{~Hz}, 50 \%$ duty scanning backlight is said to be a more suitable system in the almost all aspects including motion blur, artifact, power consumption and production cost.

\section{Conclusion}

We analyzed and determined the causes of the motion blurring phenomenon from image capturing to display terminals. Based on the proposed model, it is possible to analyze the overall frequency response characteristics. Conclusively, the $120 \mathrm{~Hz}, 50 \%$ duty scanning backlight LCDs have a relatively more suitable system than that of the $240 \mathrm{~Hz}$, $100 \%$ duty hold type LCDs, which are currently on the market. In the future, if the frequency characteristics of human visual system are added to the proposed model, it is possible to analyze the total motion blur until it is recognized in LCDs. Moreover, if we can understand the limitation of the human visual system, it can be used as a guide line when developing LCDs.

\section{References}

[1] I.H. Song, S.M. Chae, S.H. Lee, and K.I. Sohng, "Evaluation of motion blur based on the temporal frequency characteristecs of video camera and LCD TV," SID Symposium Digest of Technical papers, vol.41, Issue 1, pp.1504-1507, 2010.

[2] S.H. Lee, S.W. Jang, E.S. Kim, S.H. Lee, and K.I. Sohng, "The design of an LCD system based on the quantitative analysis for enhancement of the motion picture quality," IEICE Trans. Electron., vol.E88C, no.11, pp.2094-2098, Nov. 2005.

[3] S.K. Hong, J.H. Oh, P.Y. Park, and J.H. Park, "Motion blur reduction in LCDs," Proc. Asia Display, 2004.

[4] N. Fisekovic, T. Nauta, N.J. Cornelissen, and J. Bruinink, "Improved motion-picture quality of AM-LCDs using scanning backlight," Asia Display/IDW 01, pp.1637-1640, 2001.

[5] K. Sekiya and H. Nakamura, "Eye-trace integration effect on the perception of moving pictures and a new possibility for reducing blur on hold-type displays," Proc. SID, pp.930-933, 2002.

[6] M.A. Klompenhouwer and L.J. Velthoven, "Motion blur reduction for liquid crystal displays: motion compensated inverse filtering," Proc. SPIE-IS\&T Electronic Imaging, vol.5308, 2004. 